Скопје, Македонија

\title{
FREE POWER-SEMICOMMUTATIVE TERNARY GROUPOIDS
}

\author{
VESNA CELAKOSKA-JORDANOVA, EMILIJA CELAKOSKA, \\ AND VALENTINA MIOVSKA
}

\begin{abstract}
A description of free objects in the class $\mathcal{P}_{s c}$ of power-semicommutative ternary groupoids and a proper characterization of the class of free objects in $\mathcal{P}_{s c}$ by means of $\mathcal{P}_{s c}$-injective ternary groupoids are obtained.
\end{abstract}

\section{INTRODUCTION}

Ternary algebraic operations were introduced in the 19th century by A. Cayley. In 1928, W. Dörnte, under inspiration of Emmy Noether, introduced the notion of an $n$-ary group. Later on, in 1932, D. H. Lehmer introduced the notion of ternary algebraic system by investigating certain algebraic systems called triplexes which turn out to be commutative ternary groups ([19]). Ternary structures have many applications in different branches, such as Lie algebra theory ([22]), matrix theory ([18]), differential geometry, theoretical and mathematical physics $([17,20])$, etc. Many generalizations of binary structures to higher $n$-ary contexts are considered by many authors. Free $n$-ary algebraic structures (specially free ternary algebraic structures) are investigated, for example, in $[2,9,10,14,24]$.

Groupoids (i.e. algebras with one binary operation) with a power-property, such as power-associativity and power-commutativity, are structures that have a weak form of associativity $([12])$ or commutativity $([6])$. It seams that power-associativity have been first studied by Albert in 1948 ([1]), concerning rings. A groupoid is said to be power-associative (powercommutative) if the subgroupoid generated by any of its elements is a subsemigroup (commutative subgroupoid). The concept of power-property can be extended to $n$-ary groupoids $([11])$ and specially to ternary groupoids.

2010 Mathematics Subject Classification. Primary: 08B20, 03C05, 20 N15.

Key words and phrases. semicommutative ternary groupoid, mono-generated ternary subgroupoid, free ternary groupoid, injective semicommutative ternary groupoid. 
By a ternary groupoid we mean a nonempty set $G$ with one ternary operation [ ] : $G \times G \times G \rightarrow G$. For the notation and basic notions the reader is referred to [16] and [23].

A ternary groupoid $\boldsymbol{G}=(G,[])$ is said to be semicommutative ([3, 4, $15,21])$ if

$$
(\forall a, b, c \in G)[a b c]=[c b a] .
$$

The class $\mathcal{S}_{c}$ of all semicommutative ternary groupoids is a variety, defined by the identity $\left[x_{1} x_{2} x_{3}\right] \approx\left[x_{3} x_{2} x_{1}\right]$.

Some properties of semicommutative ternary groups are considered in [4]. Namely, a ternary group is medial if and only if it is semicommutative, and a ternary group $G$ is semicommutative if and only if there exists $a \in G$ such that $[x a y]=[y a x]$, for all $x, y \in G$. Semicommutative $n$-monoids are considered in [21].

Power-semicommutative ternary groupoids are ternary groupoids such that every ternary subgroupoid generated by any of its elements is a semicommutative ternary subgroupoid.

For example, let $G=\{0,1,2\}$ and let [ ] : $G^{3} \rightarrow G$ be a ternary operation defined by:

$$
\begin{aligned}
& {[000]=[010]=[101]=[011]=[110]=1, \quad[111]=[001]=[100]=0,} \\
& {[x y z]=2, \text { if } y=2 \text { or } z=2 \text { and }[2 y z]=1, \text { for every } y, z \in G .}
\end{aligned}
$$

Clearly, ternary subgroupoids of $\boldsymbol{G}$ generated by 0,1 and 2, respectively, are $\langle 0\rangle=\langle 1\rangle=\{0,1\}$ and $\langle 2\rangle=\{2\}$ and they are semicommutative. So, $(G,[])$ is a power-semicommutative ternary groupoid. It is not semicommutative, since, for instance, $[012]=2 \neq 1=[210]$. Also, it is not a ternary semigroup, since, for instance, $[[000] 00]=[100]=0 \neq 1=[010]=[0[000] 0]$.

In this paper we consider free objects in the class of power-semicommutative ternary groupoids.

\section{Preliminaries}

We will briefly mention the construction of the absolutely free ternary groupoid $\mathbb{T}_{X}=(T,[])$ over a nonempty set $X$, by using the construction of the absolutely free vector valued groupoid ([13]), i.e. an $(n, m)$-groupoid, for $n=3$ and $m=1$, over $X$, where $X \cap\{[]\}=\emptyset$. Let $T_{0}, T_{1}, \ldots, T_{p}, \ldots$, be a sequence of disjoint sets defined by

$$
\begin{gathered}
T_{0}=X, \quad T_{1}=\left\{\left(t_{1}, t_{2}, t_{3}\right) \mid t_{1}, t_{2}, t_{3} \in T_{0}\right\}, \\
T_{p+1}=\left\{\left(t_{1}, t_{2}, t_{3}\right) \mid t_{1}, t_{2}, t_{3} \in T_{0} \cup T_{1} \cup \cdots \cup T_{p} \wedge(\exists i \in\{1,2,3\}) t_{i} \in T_{p}\right\} .
\end{gathered}
$$

If $t \in T_{p}$, then we say that $t$ has a hierarchy $p$ and denote it by $\chi(t)=p$.

Put $T=\bigcup\left\{T_{p} \mid p \geq 0\right\}$. Define a ternary operation [] $: T \times T \times T \rightarrow T$ 
by $\left[t_{1} t_{2} t_{3}\right]=\left(t_{1}, t_{2}, t_{3}\right)$.

Then $\mathbb{T}_{X}$ is a ternary groupoid generated by $X$ that has the universal mapping property in the class of ternary groupoids over $X$, i.e. it is the absolutely free ternary groupoid over $X$. The set $X$ is the set of prime elements. Namely, an element $t \in T$ is said to be prime in $\mathbb{T}_{X}$ if $t \neq\left[t_{1} t_{2} t_{3}\right]$ for every $t_{i} \in T, i=1,2,3$ and non-prime in the opposite case. For every $t \in T$ we define the length $|t|$ of $t$, the set of subterms $P(t)$ of $t$ and the set of variables $\operatorname{var}(t)$ of $t$, by:

$t \in X \Rightarrow|t|=1, \quad t=\left[t_{1} t_{2} t_{3}\right] \Rightarrow|t|=\left|t_{1}\right|+\left|t_{2}\right|+\left|t_{3}\right| ;$

$t \in X \Rightarrow P(t)=\{t\}, \quad t=\left[t_{1} t_{2} t_{3}\right] \Rightarrow P(t)=\{t\} \cup P\left(t_{1}\right) \cup P\left(t_{2}\right) \cup P\left(t_{3}\right) ;$

$t \in X \Rightarrow \operatorname{var}(t)=\{t\}, \quad t=\left[t_{1} t_{2} t_{3}\right] \Rightarrow \operatorname{var}(t)=\operatorname{var}\left(t_{1}\right) \cup \operatorname{var}\left(t_{2}\right) \cup \operatorname{var}\left(t_{3}\right)$.

A ternary groupoid $\boldsymbol{G}$ is said to be injective if

$$
\left[a_{1} a_{2} a_{3}\right]=\left[b_{1} b_{2} b_{3}\right] \Rightarrow a_{1}=b_{1}, a_{2}=b_{2}, a_{3}=b_{3} .
$$

The absolutely free ternary groupoid $\mathbb{T}_{X}$ is injective. It is characterized by:

Theorem 1. ([16], Thm.11.2) A ternary groupoid $\boldsymbol{G}$ is absolutely free over a nonempty set $X$ if and only if the following two conditions are satisfied:

(1) $\boldsymbol{G}$ is injective;

(2) $X$ is the set of prime elements in $\boldsymbol{G}$ that generates $\boldsymbol{G}$.

Remark. We refer to this theorem as Bruck Theorem for the class of all ternary groupoids. This theorem for the binary case is stated in [5] as Lemma 1.5.

The notion of ternary groupoid powers is considered in [8] and [11]. We denote by $\mathbb{E}=(E,[])$ the ternary groupoid of terms over one-element set $\{e\}$. Note that

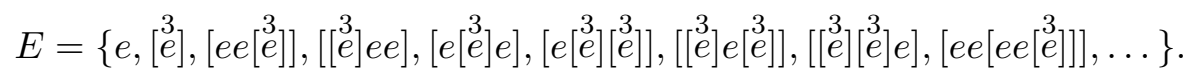

The elements of the set $E$ are called ternary groupoid powers and we denote them by $f, g, h, f_{i}, \ldots$ Every element $f \in E$ induces on a ternary groupoid $\boldsymbol{G}$ a transformation $f^{G}: G \rightarrow G$, called the interpretation of $f$ in $\boldsymbol{G}$ defined by:

$$
(\forall a \in G) \quad f^{G}(a)=\varphi_{a}(f),
$$

where $\varphi_{a}: \mathbb{E} \rightarrow \boldsymbol{G}$ is the homomorphism such that $\varphi_{a}(e)=a$. More explicitly:

$$
e(a)=a, \quad\left[f_{1} f_{2} f_{3}\right](a)=\left[f_{1}(a) f_{2}(a) f_{3}(a)\right]
$$

where $f_{i}(a)$ stands for $f_{i}^{G}(a), i=1,2,3$. Note that if $\boldsymbol{G}=\mathbb{E}$, then the above formulas, for every $g \in \mathbb{E}$, become

$$
e(g)=g, \quad\left[f_{1} f_{2} f_{3}\right](g)=\left[f_{1}(g) f_{2}(g) f_{3}(g)\right] .
$$


If one defines an operation $\circ$ on $E$ by $f \circ g=f(g)$, then one obtains an algebra $(E,[], \circ)$ with one ternary and one binary operation, [ ] and $\circ$ respectively, such that:

$$
e \circ g=g, \quad\left[f_{1} f_{2} f_{3}\right] \circ g=\left[\left(f_{1} \circ g\right)\left(f_{2} \circ g\right)\left(f_{3} \circ g\right)\right],
$$

for any $f_{1}, f_{2}, f_{3}, g \in E$. It is easily shown that $(E, \circ, e)$ is a cancellative monoid.

Proposition 1. ([8], [11]) If $t, u \in T$ and $f, g \in E$, then:

(a) $|f(t)|=|f| \cdot|t|$;

(b) $t \in P(f(t))$;

(c) $f(t)=f(u) \Rightarrow t=u$;

(d) $f(t)=g(t) \Rightarrow f=g$;

(e) $f(t)=g(u) \wedge(|f|=|g| \vee|t|=|u|) \Leftrightarrow f=g \wedge t=u$;

(f) $f(t)=g(u) \wedge|t|>|u| \Leftrightarrow(\exists ! h \in E \backslash\{e\})(t=h(u) \wedge g=f(h))$.

Definition 1. Given a ternary groupoid $\boldsymbol{G}=(G,[])$, an element $a \in G$ is said to be primitive in $\boldsymbol{G}$ if $a \neq f(c)$, for any $c \in G$ and any $f \in E \backslash\{e\} ; a$ is said to be potent in $\boldsymbol{G}$ if it is not primitive. Specially, primitive elements and potent elements in $\mathbb{T}_{X}$ are called primitive ternary terms and potent ternary terms, respectively.

Proposition 2. ([7]) The following conditions are equivalent:

(a) $v$ is primitive in $\mathbb{T}_{X}$;

(b) $(\forall u \in T)(\forall f \in E)(v=f(u) \Rightarrow f=e)$;

(c) $(\forall u \in T)(\forall f \in E)(v=f(u) \Rightarrow v=u)$.

The proof of the next proposition can be obtained in the same manner as in the binary case ([7]).

Proposition 3. For any term $t \in T$ there is a unique primitive ternary term $u \in T$ and a unique $f \in E$ such that $t=f(u)$.

In that case we call $u$ the base of $t, f$ the power of $t,|f|$ the exponent of $t$, and denote them by $\underline{t}=u, t^{\sim}=f$ and $\left|t^{\sim}\right|$, respectively.

Proposition 4. Let $t_{1}, t_{2}, t_{3}$ be ternary terms.

(a) If $t_{i}, t_{j}$ have different bases for at least one pair $\left(t_{i}, t_{j}\right)$, where $i, j \in$ $\{1,2,3\}$, then: $\left[t_{1} t_{2} t_{3}\right]=\left[t_{1} t_{2} t_{3}\right]$ and $\left(\left[t_{1} t_{2} t_{3}\right]\right)^{\sim}=e$.

(b) If $\underline{t_{1}}=\underline{t_{2}}=\underline{t_{3}}=u$, then $\underline{\left[t_{1} t_{2} t_{3}\right]}=u$ and $\left(\left[t_{1} t_{2} t_{3}\right]\right)^{\sim}=\left[t_{1}^{\sim} t_{2}^{\sim} t_{3}^{\sim}\right]$.

Proof. (a) Let $t_{1}, t_{2}, t_{3} \in T$. By Prop.3, let $t_{i}=f_{i}\left(u_{i}\right)$, where $u_{i}=\underline{t}_{i}$, $f_{i}=t_{i}^{\sim}$ for $i \in\{1,2,3\}$ and let $t=\left[t_{1} t_{2} t_{3}\right]=f(u)$, where $u=\underline{t}, f=t^{\sim}$.

Without loss of generality, let $u_{1} \neq u_{2}$. We claim that $f=e$. Suppose that $f=\left[f_{1} f_{2} f_{3}\right]$. Then $f(u)=\left[f_{1} f_{2} f_{3}\right](u)=\left[f_{1}(u) f_{2}(u) f_{3}(u)\right]$ and $f(u)=$ 
$\left[t_{1} t_{2} t_{3}\right]=\left[f_{1}\left(u_{1}\right) f_{2}\left(u_{2}\right) f_{3}\left(u_{3}\right)\right]$. By the injectivity of $\mathbb{T}_{X}, f_{i}\left(u_{i}\right)=f_{i}(u)$ and by Prop. 1 (c) it follows that $u_{i}=u, i \in\{1,2,3\}$, and this contradicts the supposition $u_{1} \neq u_{2}$. Thus, $f=e$, i.e. $\underline{t}=u=\left[t_{1} t_{2} t_{3}\right]$.

(b) Let $\underline{t_{1}}=\underline{t_{2}}=\underline{t_{3}}=u, t_{i}=f_{i}(u), i \in\{1,2,3\}$ and $t=\left[t_{1} t_{2} t_{3}\right]=$ $g(v)$. Then $g(v)=\left[f_{1}(u) f_{2}(u) f_{3}(u)\right]$. Since $v$ is a primitive term and $\left|\left[f_{1}(u) f_{2}(u) f_{3}(u)\right]\right| \geq 3$, it follows that $g \neq e$. Let $g=\left[g_{1} g_{2} g_{3}\right]$. Then

$$
\left[f_{1}(u) f_{2}(u) f_{3}(u)\right]=g(v)=\left[g_{1} g_{2} g_{3}\right](v)=\left[g_{1}(v) g_{2}(v) g_{3}(v)\right] .
$$

By the injectivity of $\mathbb{T}_{X}$ it follows that $g_{i}(v)=f_{i}(u), i \in\{1,2,3\}$. As $u$ and $v$ are primitive terms, neither $|u|>|v|$ nor $|v|>|u|$ (Prop.1 (f)). Therefore $|u|=|v|$. By Prop.1 (e) it follows that $f_{i}=g_{i}, i \in\{1,2,3\}$ and $u=v$. Thus, $\left[t_{1} t_{2} t_{3}\right]=u$. By $\left[t_{1} t_{2} t_{3}\right]=\left[f_{1}(u) f_{2}(u) f_{3}(u)\right]$ it follows that

$$
\left(\left[t_{1} t_{2} t_{3}\right]\right)^{\sim}=g=\left[f_{1}(u) f_{2}(u) f_{3}(u)\right]=\left[t_{1}^{\sim} t_{2}^{\sim} t_{3}^{\sim}\right] .
$$

\section{A construction of CAnonical objects in $\mathcal{P}_{s c}$}

The ternary subgroupoid of a ternary groupoid $\boldsymbol{G}=(G,[])$ generated by an element $a \in G$ will be denoted by $\langle a\rangle$ and called the mono-generated ternary subgroupoid of $\boldsymbol{G}$ with the generator $a$. A ternary groupoid $\boldsymbol{G}$ is said to be power-semicommutative if every mono-generated ternary subgroupoid of $\boldsymbol{G}$ is semicommutative. We denote the class of power-semicommutative ternary groupoids by $\mathcal{P}_{s c}$. Mono-generated ternary subgroupoids of a ternary groupoid can be characterized as:

$$
(\forall a \in G)\langle a\rangle=\{f(a): f \in E\} .
$$

This enables to obtain an axiom system for $\mathcal{P}_{s c}$.

Proposition 5. A ternary groupoid $\boldsymbol{G}$ is power-semicommutative if and only if

$$
(\forall a \in G)\left(\forall f_{1}, f_{2}, f_{3} \in E\right)\left[f_{1}(a) f_{2}(a) f_{3}(a)\right]=\left[f_{3}(a) f_{2}(a) f_{1}(a)\right] .
$$

As a consequence of Proposition 5 it follows that the class of powersemicommutative ternary groupoids $\mathcal{P}_{s c}$ is a variety of ternary groupoids defined by the system of identities

$$
\left\{\left[f_{1}(x) f_{2}(x) f_{3}(x)\right] \approx\left[f_{3}(x) f_{2}(x) f_{1}(x)\right]: f_{1}, f_{2}, f_{3} \in E\right\} .
$$

We are looking for a canonical groupoid in $\mathcal{P}_{s c}$.

Definition 2. A groupoid $\boldsymbol{R}=\left(R,[]^{*}\right)$ is called a canonical powersemicommutative ternary groupoid if the following conditions are satisfied:

(C0) $X \subseteq R \subseteq T$ and $t \in R \Rightarrow P(t) \subseteq R$; 
(C1) $t_{1}, t_{2}, t_{3},\left[t_{1} t_{2} t_{3}\right] \in R \Rightarrow\left[t_{1} t_{2} t_{3}\right]^{*}=\left[t_{1} t_{2} t_{3}\right]$;

(C2) $\boldsymbol{R}$ is a free ternary groupoid in $\mathcal{P}_{s c}$ over $X$ (i.e. $\mathcal{P}_{s c}$-free over $X$ ).

Further on we will use an ordering on $T$ assuming that $X$ is a linearly ordered set $([9])$, where the ordering relation is denoted by $\leq$. Ternary terms are ordered so that a ternary term of a particular hierarchy comes before any term of a higher hierarchy and among ternary terms of equal hierarchy lexicographical ordering is used, with ternary terms earlier in the lexicographical ordering coming first. The precise definition is given below.

Definition 3. Let $X$ be a linearly ordered set and $t, u \in T$.

(i) $t, u \in X \Rightarrow(t \leq u$ in $T \Leftrightarrow t \leq u$ in $X)$;

(ii) $\chi(t)<\chi(u) \Rightarrow t<u$;

(iii) If $\chi(t)=\chi(u) \geq 1$ and $t \neq u$, where $t=\left[t_{1} t_{2} t_{3}\right], u=\left[u_{1} u_{2} u_{3}\right]$, then $t<u$ if and only if $\left[t_{1}<u_{1} \vee\left(t_{1}=u_{1} \wedge t_{2}<u_{2}\right) \vee\left(t_{1}=u_{1} \wedge t_{2}=u_{2} \wedge t_{3}<u_{3}\right)\right]$.

Then $\leq$ is a linear ordering of $T$.

We will use the free semicommutative ternary groupoid constructed as follows ([9]). Define a subset $T_{s c}$ of $T$ by

$$
T_{s c}=X \cup\left\{t \in T \backslash X:\left[t_{1} t_{2} t_{3}\right] \in P(t) \Rightarrow t_{1} \leq t_{3}\right\}
$$

and an operation [ $]^{\prime}$ on $T_{s c}$ by $\left[t_{1} t_{2} t_{3}\right]^{\prime}=\left[t_{1} t_{2} t_{3}\right]$ if $t_{1} \leq t_{3}$, and, $\left[t_{1} t_{2} t_{3}\right]^{\prime}=$ $\left[t_{3} t_{2} t_{1}\right]$ if $t_{3}<t_{1}$, for every $t_{1}, t_{2}, t_{3} \in T_{s c}$. Then $\left.\mathbb{T}_{s c}=\left(T_{s c} \text {, [ ] }\right]^{\prime}\right)$ is a canonical semicommutative ternary groupoid over $X$.

Specially, $\mathbb{E}_{s c}=\left(E_{s c},[]^{\prime}\right)$ is a canonical semicommutative ternary groupoid over $e$, where $E_{s c}=\{e\} \cup\left\{f \in E \backslash\{e\}:\left[f_{1} f_{2} f_{3}\right] \in P(f) \Rightarrow f_{1} \leq f_{3}\right\}$.

Let $\psi: E \rightarrow E_{s c}$ be a homomorphism from $\mathbb{E}$ into $\mathbb{E}_{s c}$ such that $\psi(e)=e$. For every $f \in E$ put $\psi(f)=\bar{f}$. If $f=\left[f_{1} f_{2} f_{3}\right] \in E$, we obtain that $\left.\overline{\left[f_{1} f_{2} f_{3}\right.}\right]=\left[\overline{f_{1}} \overline{f_{2}} \overline{f_{3}}\right]^{\prime}$. For instance, if $f=\left[\begin{array}{l}3 \\ ]\end{array}\right]$, then $\left.\psi(f)=\overline{[e}\right]=\left[\begin{array}{l}3 \\ e\end{array}\right]$, but if

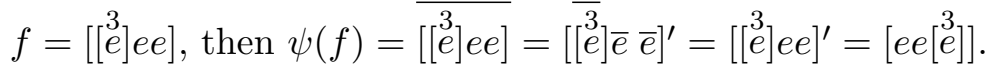

Clearly, if $f \in E_{s c}$, then $f=\bar{f}$.

A term $t \in T$ is said to be

(1) laterally order-regular if $t \in X$ or $\left(t=\left[t_{1} t_{2} t_{3}\right] \wedge t_{1} \leq t_{3}\right)$.

Specially, a ternary groupoid power $f$ is laterally order-regular if and only if $f=e$ or $\left(f=\left[f_{1} f_{2} f_{3}\right] \wedge f_{1} \leq f_{3}\right)$.

(2) order-regular if $t \in X$ or $\left(t=\left[t_{1} t_{2} t_{3}\right] \wedge t_{1} \leq t_{2} \leq t_{3}\right)$.

Specially, a ternary groupoid power $f$ is order-regular if and only if $f=e$ or $\left(f=\left[f_{1} f_{2} f_{3}\right] \wedge f_{1} \leq f_{2} \leq f_{3}\right)$.

By induction on the length of $f$, one can show the following two statements as in the binary case $([6])$. 
Lemma 1. Let $f, g, h \in E$.

(a) If $f<g$, then $f(h)<g(h)$.

(b) $f(h)$ is laterally order-regular (order-regular) if and only if $f$ and $h$ are laterally order-regular (order-regular).

To obtain a description of the canonical objects in $\mathcal{P}_{s c}$, define the set

$$
R=\left\{t \in T: u \in P(t) \Rightarrow u^{\sim} \in E_{s c}\right\} .
$$

In other words, a term $t \in R$ if and only if the power of any subterm $u$ of $t$ is laterally order-regular, i.e. if $u \in X$ or $\left(u=\left[u_{1} u_{2} u_{3}\right] \Rightarrow u_{1}^{\sim} \leq u_{3}^{\sim}\right)$.

From (1) we obtain the following consequences:

(a) $X \subset R \subset T$;

(b) $\left[t_{1} t_{2} t_{3}\right] \in R \Rightarrow t_{1}, t_{2}, t_{3} \in R$;

(c) $t_{1}, t_{2}, t_{3} \in R \Rightarrow\left\{\left[t_{1} t_{2} t_{3}\right] \notin R \Leftrightarrow \underline{t_{1}}=\underline{t_{2}}=\underline{t_{3}} \wedge t_{3}^{\sim}<t_{1}^{\sim}\right\}$;

(d) $t_{1}, t_{2}, t_{3} \in T \Rightarrow\left\{\left[t_{1} t_{2} t_{3}\right] \in R \Leftrightarrow \overline{t_{1}}, t_{2}, t_{3} \in \bar{R} \wedge\right.$

$$
\left.\wedge\left((\exists i, j \in\{1,2,3\}) \underline{t_{i}} \neq \underline{t_{j}} \vee\left(\underline{t_{1}}=\underline{t_{2}}=\underline{t_{3}} \wedge t_{1}^{\sim} \leq t_{3}^{\sim}\right)\right)\right\} .
$$

Define an operation [ ]* on $R$ by:

$$
t_{1}, t_{2}, t_{3} \in R \Rightarrow\left[t_{1} t_{2} t_{3}\right]^{*}= \begin{cases}{\left[t_{1} t_{2} t_{3}\right],} & \text { if }\left[t_{1} t_{2} t_{3}\right] \in R \\ {\left[t_{3} t_{2} t_{1}\right],} & \text { if } \underline{t_{1}}=\underline{t_{2}}=\underline{t_{3}} \wedge t_{3}^{\sim}<t_{1}^{\sim} .\end{cases}
$$

Theorem 2. The groupoid $\boldsymbol{R}=\left(R,[]^{*}\right)$ is a canonical power-semicommutative ternary groupoid over $X$.

Proof. From $(c),(d)$ and $(2)$ it follows that $\boldsymbol{R}=\left(R,[]^{*}\right)$ is a ternary groupoid. It is clear that $X$ is the set of primes in $\boldsymbol{R}$ and generates $\boldsymbol{R}$. To prove that $\boldsymbol{R} \in \mathcal{P}_{s c}$, it suffices to show that every mono-generated ternary subgroupoid of $\boldsymbol{R}$ is semicommutative. For that reason we will use the interpretation of $f \in E$ in $\boldsymbol{R}$, denoted by $f_{*}(t)$ :

$$
(\forall t \in R)\left(e_{*}(t)=t \wedge\left[f_{1} f_{2} f_{3}\right]_{*}(t)=\left[f_{1 *}(t) f_{2 *}(t) f_{3 *}(t)\right]^{*}\right)
$$

By induction on the length of $f(|f|=2 k-1, k \in \mathbb{N})$ and using Lemma 1 (a), one can show that for every $t \in R$ :

1) $\left.f \in E_{s c} \Rightarrow f_{*}(t)=f(t) ; \quad 2\right) f \in E \Rightarrow f_{*}(t)=\bar{f}(t)$.

Namely, for 1): if $k=1$, then $f=e$ and $e_{*}(t)=t=e(t)$. Assume that the claim is true for all the powers $f$ up to some length $2 k-1$ and let $f=\left[f_{1} f_{2} f_{3}\right] \in E_{s c}$ be such that $|f|=2 k+1$. Then $\left|f_{i}\right| \leq 2 k-1$, $i \in\{1,2,3\}$ and thus

$$
\begin{aligned}
f_{*}(t) & =\left[f_{1} f_{2} f_{3}\right]_{*}(t)=\left[f_{1 *}(t) f_{2 *}(t) f_{3 *}(t)\right]^{*}= \\
& =\left[f_{1}(t) f_{2}(t) f_{3}(t)\right]^{*}=\left[f_{1} f_{2} f_{3}\right]^{\prime}(t)=f(t) .
\end{aligned}
$$


For 2): let $f \in E$. If $f \in E_{s c}$, then by (a), $f_{*}(t)=f(t)=\bar{f}(t)$. Therefore, let $f \in E \backslash E_{s c}$. For the shortest $f \in E \backslash E_{s c}$, i.e. for $f=\left[\left[{ }^{3}\right] e e\right]$, we have

$$
\begin{aligned}
f_{*}(t) & =\left[\left[\begin{array}{l}
3 \\
e
\end{array}\right] e e\right]_{*}(t)=\left[\left[\begin{array}{l}
3 \\
e
\end{array}\right]_{*}(t) e_{*}(t) e_{*}(t)\right]^{*}= \\
& =\left[\left[{ }^{3} e\right](t) e(t) e(t)\right]^{*}=\left[\left[\begin{array}{l}
3 \\
e
\end{array}\right] e e\right]^{\prime}(t)=\left[e e\left[\begin{array}{l}
3 \\
e
\end{array}\right]\right](t)=\bar{f}(t) .
\end{aligned}
$$

Assume that the claim is true up to some $f$ with $|f|=2 k-1$ and let $f=\left[f_{1} f_{2} f_{3}\right] \in E \backslash E_{s c}$ be with $|f|=2 k+1$. Then $\left|f_{i}\right| \leq 2 k-1$, $i \in\{1,2,3\}$ and thus

$$
\begin{aligned}
f_{*}(t) & =\left[f_{1} f_{2} f_{3}\right]_{*}(t)=\left[f_{1 *}(t) f_{2 *}(t) f_{3 *}(t)\right]^{*}= \\
& =\left[\overline{f_{1}}(t) \overline{f_{2}}(t) \overline{f_{3}}(t)\right]^{*}=\left[\overline{f_{1}} \overline{f_{2}} \overline{f_{3}}\right]^{\prime}(t)=\overline{\left[f_{1} f_{2} f_{3}\right]}(t)=\bar{f}(t) .
\end{aligned}
$$

Now we will show that every mono-generated ternary subgroupoid $\langle t\rangle_{*}$, $t \in R$, is a semicommutative ternary subgroupoid of $\boldsymbol{R}$, i.e. $\boldsymbol{R} \in \mathcal{P}_{s c}$.

Namely, let $t \in R$. Then by 2$), f_{*}(t)=\bar{f}(t)$ for every $f \in E$, i.e. $\left\{f_{*}(t): f \in E\right\} \subseteq\left\{f_{*}(t): f \in E_{s c}\right\}$. Since $E_{s c} \subset E$ and by 1$)$, it follows that the inverse inclusion is true as well. Therefore, $\left\{f_{*}(t): f \in E\right\}=$ $\left\{f_{*}(t): f \in E_{s c}\right\}$. Using the fact that for any $t \in R,\langle t\rangle_{*}=\left\{f_{*}(t): f \in E\right\}$ one obtains that $\langle t\rangle_{*} \in \mathcal{S}_{c}$.

It remains to show that the ternary groupoid $\boldsymbol{R}$ has the universal mapping property for $\mathcal{P}_{s c}$ over $X$.

Let $\boldsymbol{G}=\left(G,[]^{\prime \prime}\right) \in \mathcal{P}_{s c}, \lambda: X \rightarrow G$ be any mapping and $\varphi: \mathbb{T}_{X} \rightarrow \boldsymbol{G}$ be the homomorphism that is an extension of $\lambda$. If $t_{1}, t_{2}, t_{3} \in R$ and $\left[t_{1} t_{2} t_{3}\right] \in$ $R$, then $\varphi\left(\left[t_{1} t_{2} t_{3}\right]^{*}\right)=\varphi\left(\left[t_{1} t_{2} t_{3}\right]\right)=\left[\varphi\left(t_{1}\right) \varphi\left(t_{2}\right) \varphi\left(t_{3}\right)\right]^{\prime \prime}$. If $\left[t_{1} t_{2} t_{3}\right] \notin R$, then $\underline{t_{1}}=\underline{t_{2}}=\underline{t_{3}}$ and $t_{3}^{\sim}<t_{1}^{\sim}$. Let $\underline{t_{i}}=u$ and $t_{i}=f_{i}(u)$ for some $f_{i} \in E$, $i \in\{1,2,3\}$, i.e. $t_{i} \in\langle u\rangle_{*}$. It is clear that $\varphi\left(t_{i}\right) \in\langle\varphi(u)\rangle$ and so

$$
\varphi\left(\left[t_{1} t_{2} t_{3}\right]^{*}\right)=\varphi\left(\left[t_{1} t_{2} t_{3}\right]\right)=\left[\varphi\left(t_{3}\right) \varphi\left(t_{2}\right) \varphi\left(t_{1}\right)\right]^{\prime \prime}=\left[\varphi\left(t_{1}\right) \varphi\left(t_{2}\right) \varphi\left(t_{3}\right)\right]^{\prime \prime},
$$

since $\boldsymbol{G} \in \mathcal{P}_{s c}$. Therefore, $\left.\varphi\right|_{R}: \boldsymbol{R} \rightarrow \boldsymbol{G}$ is a homomorphism that extends $\lambda$. Hence, $\boldsymbol{R}$ is $\mathcal{P}_{s c}$-free groupoid over $X$ that has the properties $(\mathrm{C} 0)$ and (C1). Thus, (1) and (2) give a description of the canonical objects in the variety of power-semicommutative ternary groupoids.

\section{A Characterization of $\mathcal{P}_{s c}$-FreE ternary Groupoids}

We will define a subclass of the class $\mathcal{P}_{s c}$ that is larger than the class of $\mathcal{P}_{s c}$-free ternary groupoids called the class of $\mathcal{P}_{s c}$-injective ternary groupoids in order to obtain a characterization of free objects in $\mathcal{P}_{s c}$. For that reason we will use the properties of the corresponding $\mathcal{P}_{s c}$-canonical ternary groupoid $\boldsymbol{R}=\left(R,[]^{*}\right)$ related to the elements of $R$ that are not prime in $\boldsymbol{R}$. The class of $\mathcal{P}_{s c}$-injective ternary groupoids will be successfully defined if the following two conditions are satisfied: 
1) the class of $\mathcal{P}_{s c}$-injective ternary groupoids should enable a characterization of $\mathcal{P}_{s c}$-free ternary groupoids analogously as in Theorem 1;

2) the class of $\mathcal{P}_{s c}$-free ternary groupoids has to be a proper subclass of the class of $\mathcal{P}_{s c}$-injective ternary groupoids.

Therefore, we will state some properties that are related to the nonprime elements in $\left(R,[]^{*}\right)$ that are necessary for defining the notion of $\mathcal{P}_{s c}$-injectivity.

Note that $t \in R$ is primitive in $\boldsymbol{R}$ if and only if $t$ is primitive in $\mathbb{T}_{X}$.

Similarly as in Prop. 3 and Prop. 4 one obtains:

Lemma 2. Let $t_{1}, t_{2}, t_{3} \in R$.

a) For any $t \in R$ there is a unique primitive term $u \in R$ and a unique $f \in E_{\text {sc }}$, such that $t=f_{*}(u)$. Here we also write, $\underline{t}=u$ and $t^{\sim}=f$.

b) If $t_{i}, t_{j}$ have different bases for at least one pair $t_{i}, t_{j}, i, j \in\{1,2,3\}$, then: $\left[t_{1} t_{2} t_{3}\right]=\left[t_{1} t_{2} t_{3}\right]$ and $\left(\left[t_{1} t_{2} t_{3}\right]\right)^{\sim}=e$.

c) If $\underline{t_{1}}=\underline{t_{2}}=\underline{t}_{3}=u$, then: $\left[t_{1} t_{2} t_{3}\right]=u$ and $\left(\left[t_{1} t_{2} t_{3}\right]\right)^{\sim}=\left[t_{1}^{\sim} t_{2}^{\sim} t_{3}^{\sim}\right]^{\prime}$.

If a term $t \in R$ is not prime in $\boldsymbol{R}$, then $t=\left[t_{1} t_{2} t_{3}\right]^{*}$ for $t_{1}, t_{2}, t_{3} \in R$. In that case we say that $\left(t_{1}, t_{2}, t_{3}\right)$ is a triple of divisors of $t$ in $\boldsymbol{R}$, where $t_{1}$ is the left, $t_{2}$ is the middle and $t_{3}$ is the right divisor of $t$; we write $\left(t_{1}, t_{2}, t_{3}\right) \mid t$.

Proposition 6. Let $t$ be a non-prime element in $\boldsymbol{R}$. Then there is a unique triple $\left(t_{1}, t_{2}, t_{3}\right)$ of $R^{3}$ such that $t=\left[t_{1} t_{2} t_{3}\right]^{*}=\left[t_{1} t_{2} t_{3}\right]$.

We call $\left[t_{1} t_{2} t_{3}\right]$ the canonical triple of divisors of $t$ in $\boldsymbol{R}$. This triple coincides with a triple of divisors in $\mathbb{T}_{X}$.

Proof. If $t \in R \backslash X$ then $t \in T \backslash X$ and thus $t=\left[t_{1} t_{2} t_{3}\right]$ for a unique triple $\left(t_{1}, t_{2}, t_{3}\right)$ of $T^{3}$. Since $\left[t_{1} t_{2} t_{3}\right] \in R$ it follows that $t_{1}, t_{2}, t_{3} \in R$ and thus $t=\left[t_{1} t_{2} t_{3}\right]^{*}=\left[t_{1} t_{2} t_{3}\right]$.

Proposition 7. If $u_{i}, v_{i} \in R, i \in\{1,2,3\}$, are such that $\left[u_{1} u_{2} u_{3}\right]^{*}=$ $\left[v_{1} v_{2} v_{3}\right]^{*}$, and $\left[u_{1} u_{2} u_{3}\right] \notin R$ or $\left[v_{1} v_{2} v_{3}\right] \notin R$, then $\underline{u_{i}}=\underline{v_{i}}, i \in\{1,2,3\}$, and $u_{2}^{\sim}=v_{2}^{\sim},\left\{u_{1}^{\sim}, u_{3}^{\sim}\right\}=\left\{v_{1}^{\sim}, v_{3}^{\sim}\right\}$.

Proof. If $\left[u_{1} u_{2} u_{3}\right] \notin R$ and $\left[v_{1} v_{2} v_{3}\right] \in R$, then by the consequence $(c)$ of (1) (Definition of $R$ ), $\underline{u_{1}}=\underline{u_{2}}=\underline{u_{3}}=\alpha, u_{3}^{\tilde{r}}<u_{1}^{\tilde{1}}$, where $\alpha$ is a primitive term in $\boldsymbol{R}$, and thus there are $f_{i} \in E_{s c}$ such that $u_{i}=f_{i}(\alpha)$, where $f_{i}=u_{i}^{\sim}, i \in\{1,2,3\}$. Therefore, the equation $\left[u_{1} u_{2} u_{3}\right]^{*}=\left[v_{1} v_{2} v_{3}\right]^{*}$ becomes $\left[u_{3} u_{2} u_{1}\right]=\left[v_{1} v_{2} v_{3}\right]$ in $\mathbb{T}_{X}$, i.e. $\left[f_{3}(\alpha) f_{2}(\alpha) f_{1}(\alpha)\right]=\left[v_{1} v_{2} v_{3}\right]$, that implies $f_{3}(\alpha)=v_{1}, f_{2}(\alpha)=v_{2}, f_{1}(\alpha)=v_{3}$ and $\underline{v_{1}}=\underline{v_{2}}=\underline{v_{3}}=\alpha, v_{1}^{\sim}=f_{3}$, $v_{2}^{\sim}=f_{2}, v_{3}^{\tilde{z}}=f_{1}$. Thus, $\underline{u_{i}}=\underline{v_{i}}, i \in\{1,2,3\}$, and, $u_{1}^{\sim}=v_{3}^{\tilde{r}}, u_{2}^{\tilde{2}}=v_{2}^{\tilde{2}}$, $u_{3}^{\sim}=v_{1}^{\sim}$. The same conclusion is true when $\left[u_{1} u_{2} u_{3}\right] \in R$ and $\left[v_{1} v_{2} v_{3}\right] \notin R$.

If $\left[u_{1} u_{2} u_{3}\right] \notin R$ and $\left[v_{1} v_{2} v_{3}\right] \notin R$, then $\underline{u_{1}}=\underline{u_{2}}=\underline{u_{3}}=\alpha, u_{3}^{\tilde{r}}<u_{1}^{\sim}$, 
where $\alpha$ is a primitive element in $\boldsymbol{R}$ and $\underline{v_{1}}=\underline{v_{2}}=\underline{v_{3}}=\beta, v_{3}^{\sim}<v_{1}^{\sim}$, where $\beta$ is a primitive element in $\boldsymbol{R}$. So, we can write $u_{i}=u_{i}^{\sim}(\alpha), v_{i}=v_{i}^{\sim}(\beta), i \in$ $\{1,2,3\}$. The equation $\left[u_{1} u_{2} u_{3}\right]^{*}=\left[v_{1} v_{2} v_{3}\right]^{*}$ becomes $\left[u_{3} u_{2} u_{1}\right]=\left[v_{3} v_{2} v_{1}\right]$ in $\mathbb{T}_{X}$ and thus $u_{i}=v_{i}$, i.e. $u_{i}^{\sim}(\alpha)=v_{i}^{\sim}(\beta)$. This implies that $u_{i}^{\sim}=v_{i}^{\sim}$, $\alpha=\beta$, i.e. $\underline{u_{i}}=\underline{v_{i}}, i \in\{1,2,3\}$ and $u_{1}^{\sim}=v_{1}^{\sim}, u_{3}^{\sim}=v_{3}^{\tilde{3}}$.

Therefore, $\left[u_{1} u_{2} u_{3}\right]^{*}=\left[v_{1} v_{2} v_{3}\right]^{*}$ and $\left(\left[u_{1} u_{2} u_{3}\right] \notin R\right.$ or $\left.\left[v_{1} v_{2} v_{3}\right] \notin R\right)$ implies that $\underline{u_{i}}=\underline{v_{i}}, i \in\{1,2,3\}$, and $u_{2}^{\sim}=v_{2}^{\sim},\left\{u_{1}^{\sim}, u_{3}^{\sim}\right\}=\left\{v_{1}^{\sim}, v_{3}^{\sim}\right\}$.

By Prop.6 and Prop.7 one obtains the following statement.

If $\left[u_{1} u_{2} u_{3}\right]^{*}=\left[v_{1} v_{2} v_{3}\right]^{*}$, then:

1) $u_{1}=v_{1}, u_{2}=v_{2}, u_{3}=v_{3}$, when $\left[u_{1} u_{2} u_{3}\right],\left[v_{1} v_{2} v_{3}\right] \in R$ or $\left[u_{1} u_{2} u_{3}\right]$, $\left[v_{1} v_{2} v_{3}\right] \notin R$

2) $u_{1}=v_{3}, u_{3}=v_{1}$, when $\left(\left[u_{1} u_{2} u_{3}\right] \notin R\right.$ and $\left.\left[v_{1} v_{2} v_{3}\right] \in R\right)$ or $\left(\left[u_{1} u_{2} u_{3}\right] \in\right.$ $R$ and $\left.\left[v_{1} v_{2} v_{3}\right] \notin R\right)$.

In that case $\underline{u_{i}}=\underline{v_{i}}$ and $u_{i}^{\sim}=v_{i}^{\tilde{i}}$, for $i=1,2,3$.

The pertinent result in this regard is the following

Corollary 1. Let $w \in R$ be a non-prime element in $\boldsymbol{R}$, i.e. $w=\left[v_{1} v_{2} v_{3}\right]^{*}$.

a) If $w$ is primitive in $\boldsymbol{R}$, then $\left(v_{1}, v_{2}, v_{3}\right)$ is the unique triple of divisors of $w$ (the canonical one).

b) If $w$ is a potent term in $\boldsymbol{R}$, i.e. $\underline{v_{1}}=\underline{v_{2}}=\underline{v_{3}}$, then $\left(v_{3}, v_{2}, v_{1}\right)$ is the unique triple of divisors in $\boldsymbol{R}$ when $\overline{v_{1}^{\sim}}=\overline{v_{3}^{\sim}}$, and $\left(v_{1}, v_{2}, v_{3}\right),\left(v_{3}, v_{2}, v_{1}\right)$ are the only two triples of divisors of $w$ when $v_{1}^{\sim}>v_{3}^{\sim}$.

Now we will introduce the concept of injectivity in $\mathcal{P}_{s c}$.

Definition 4. A ternary groupoid $\boldsymbol{H}=(H,[])$ is said to be $\mathcal{P}_{s c}$-injective if the following conditions are satisfied.

(I0) $\boldsymbol{H} \in \mathcal{P}_{s c}$.

(I1) For any $a \in H$ there is a unique primitive element $b \in H$ and a unique $f \in E_{s c}$ such that $a=f(b)$.

(Then $b$ is called the base of $a$ and $f$ the power of $a$; we write: $\underline{a}=b$, $a^{\sim}=f$, respectively).

(I2) Let $a_{1}, a_{2}, a_{3} \in H$. If $\underline{a_{i}} \neq a_{j}$ for at least one pair $\left(a_{i}, a_{j}\right), i, j \in$ $\{1,2,3\}$, then $\left[a_{1} a_{2} a_{3}\right]=\left[a_{1} a_{2} \overline{a_{3}}\right]$ and $\left(\left[a_{1} a_{2} a_{3}\right]\right)^{\sim}=e$.

If $\underline{a_{i}}=b, i=1,2,3$, then $\left[a_{1} a_{2} a_{3}\right]=b$ and $\left(\left[a_{1} a_{2} a_{3}\right]\right)^{\sim}=\left[a_{1}^{\sim} a_{2}^{\sim} a_{3}^{\sim}\right]$ when $a_{1}^{\sim} \leq \overline{a_{3}} \tilde{3}$ or $\left(\left[a_{1} a_{2} a_{3}\right]\right)^{\sim}=\left[\overline{\left.a_{3}^{\tilde{a}} a_{2}^{\tilde{a}} a_{1}^{\sim}\right]}\right.$ when $a_{3}^{\tilde{3}} \leq a_{1}^{\tilde{1}}$.

(I3) Let $a \in H$ be a non-prime element in $\boldsymbol{H}$, i.e. $a=\left[a_{1} a_{2} a_{3}\right]$.

If $a$ is primitive in $\boldsymbol{H}$, then $\left(a_{1}, a_{2}, a_{3}\right)$ is the unique triple of divisors of $a$.

If $a$ is potent in $\boldsymbol{H}$, i.e. $\underline{a_{1}}=\underline{a_{2}}=\underline{a_{3}}$, then:

a) $\left(a_{1}, a_{2}, a_{3}\right)$ is the unique triple of divisors of $a$ when $a_{1}^{\sim}=a_{3}^{\sim}$; 
b) $\left(a_{1}, a_{2}, a_{3}\right),\left(a_{3}, a_{2}, a_{1}\right)$ are the unique two triples of divisors when $a_{1}^{\sim}>a_{3}^{\sim}$.

By Theorem 2, Lemma 2 and Cor.1, the ternary groupoid $\boldsymbol{R}$ satisfies the conditions $(I 0)-(I 3)$. Since any $\mathcal{P}_{s c}$-free ternary groupoid over $X$ is isomorphic to $\boldsymbol{R}$, one obtains that:

Proposition 8. Every $\mathcal{P}_{\text {sc }}$-free ternary groupoid is $\mathcal{P}_{\text {sc-injective. }}$

In order to prove Bruck theorem for the class $\mathcal{P}_{s c}$ we will use the following Lemma 3 (that can be proved in a similar way as in [9], Lemma 3.1).

Lemma 3. Let $\boldsymbol{H}=(H,[])$ be a $\mathcal{P}_{\text {sc-injective ternary groupoid such that }}$ the set $P$ of prime elements in $\boldsymbol{H}$ is nonempty and generates $\boldsymbol{H}$. If $C_{0}=P, C_{1}=\left[C_{0} C_{0} C_{0}\right], C_{k+1}=\left\{a \in H \backslash P:\left(d_{1}, d_{2}, d_{3}\right) \mid a \Rightarrow\right.$ $\left.\Rightarrow\left\{d_{1}, d_{2}, d_{3}\right\} \subset C_{0} \cup \cdots \cup C_{k} \wedge\left\{d_{1}, d_{2}, d_{3}\right\} \cap C_{k} \neq \varnothing\right\}$, then $H=\bigcup\left\{C_{k}: k \geq 0\right\}$, where $C_{k} \neq \varnothing$ for any $k \geq 0$ and $C_{i} \cap C_{j}=\varnothing$ for $i \neq j$.

Theorem 3. (Bruck theorem for $\mathcal{P}_{s c}$ ) A power-semicommutative ternary groupoid $\boldsymbol{H}=(H,[])$ is $\mathcal{P}_{\text {sc }}$-free if and only if

(i) $\boldsymbol{H}$ is $\mathcal{P}_{\text {sc-injective }}$

(ii) The set $P$ of prime elements in $\boldsymbol{H}$ is nonempty and generates $\boldsymbol{H}$.

Then the set of primes in $\boldsymbol{H}$ is the unique $\mathcal{P}_{\text {sc }}$-free generating set of $\boldsymbol{H}$.

Proof. If $\boldsymbol{H}$ is $\mathcal{P}_{s c}$-free, then by Prop.8 and the fact that $\boldsymbol{H}$ is isomorphic to $\boldsymbol{R}$ it follows that $(i)$ and $(i i)$ are fulfilled.

Conversely, let $(i)$ and (ii) hold. By Lemma 3, $H=\bigcup\left\{C_{k}: k \geq 0\right\}$. To show that $\boldsymbol{H}$ is $\mathcal{P}_{s c}$-free over the set $P$, we need to show that it has the universal mapping property for $\mathcal{P}_{s c}$ over $P$. For this reason let $\boldsymbol{G}=$ $\left(G,[]^{\prime}\right)$ be a power-semicommutative ternary groupoid and $\lambda: P \rightarrow G$ be a mapping. Define a sequence of mappings $\varphi_{k}: C_{k} \rightarrow G$ inductively by: $\varphi_{0}=\lambda$ and let $\varphi_{i}: C_{i} \rightarrow G$ be defined for every $i \leq k$. If $a \in C_{k+1}$ and $\left(d_{1}, d_{2}, d_{3}\right)$ is a triple of divisors of $a$ where $d_{1} \in C_{p}, d_{2} \in C_{q}, d_{3} \in C_{r}$, then $p, q, r \leq k$. Putting

$$
\varphi_{k+1}\left(\left[d_{1} d_{2} d_{3}\right]\right)=\left[\varphi_{k}\left(d_{1}\right) \varphi_{k}\left(d_{2}\right) \varphi_{k}\left(d_{3}\right)\right]^{\prime}
$$

we obtain that $\varphi=\cup\left\{\varphi_{i}: i \geq 0\right\}$ is a mapping from $H$ into $G$ that is a homomorphism. Therefore:

$$
\varphi(a)=\left[\varphi_{k}\left(d_{1}\right) \varphi_{k}\left(d_{2}\right) \varphi_{k}\left(d_{3}\right)\right]^{\prime}=\left[\varphi\left(d_{1}\right) \varphi\left(d_{2}\right) \varphi\left(d_{3}\right)\right]^{\prime} .
$$

Since $\boldsymbol{H}$ is $\mathcal{P}_{s c}$-injective it follows that $\left(d_{3}, d_{2}, d_{1}\right)$ is a triple of divisors of $a$ as well and that

$$
\varphi\left(\left[d_{3} d_{2} d_{1}\right]\right)=\left[\varphi\left(d_{3}\right) \varphi\left(d_{2}\right) \varphi\left(d_{1}\right)\right]^{\prime}=\left(\boldsymbol{G} \in \mathcal{P}_{s c}\right)=
$$




$$
=\left[\varphi\left(d_{1}\right) \varphi\left(d_{2}\right) \varphi\left(d_{3}\right)\right]^{\prime}=\varphi\left(\left[d_{1} d_{2} d_{3}\right]\right) .
$$

Thus $\varphi$ is a well defined mapping and a homomorphism. Hence, $\boldsymbol{H}$ is $\mathcal{P}_{s c}$-free over $P$.

The next example shows that there is a $\mathcal{P}_{s c}$-injective ternary groupoid that is not free in $\mathcal{P}_{s c}$, i.e. the class of free ternary groupoids in $\mathcal{P}_{s c}$ is a proper subclass of the class of $\mathcal{P}_{s c}$-injective ternary groupoids.

Example. Let $X$ be an infinite countable set and $\boldsymbol{R}=\left(R,[]^{*}\right)$ be the canonical power-semicommutative ternary goupoid over $X$. Define two subsets $H \subset R$ and $D \subset H^{3}$ as follows:

$H=\{t \in R:|\operatorname{var}(t)|=1\}$, $D=\left\{\left(t_{1}, t_{2}, t_{3}\right) \in H^{3}: \operatorname{var}\left(t_{i}\right) \neq \operatorname{var}\left(t_{j}\right)\right.$ for at least one pair of $\left.i, j \in\{1,2,3\}\right\}$ and define a relation $\theta$ in $D$ by:

$$
\left(t_{1}, t_{2}, t_{3}\right) \theta\left(u_{1}, u_{2}, u_{3}\right) \Leftrightarrow t_{2}=u_{2} \wedge\left\{t_{1}, t_{3}\right\}=\left\{u_{1}, u_{3}\right\} .
$$

It is clear that $\theta$ is an equivalence relation in $D$. If $\operatorname{var}\left(t_{1}\right) \neq \operatorname{var}\left(t_{3}\right)$, then the equivalence class $\left(t_{1}, t_{2}, t_{3}\right)^{\theta}=\left\{\left(t_{1}, t_{2}, t_{3}\right),\left(t_{3}, t_{2}, t_{1}\right)\right\}$ and if $\operatorname{var}\left(t_{1}\right)=$ $\operatorname{var}\left(t_{3}\right)$, then $\left(t_{1}, t_{2}, t_{3}\right)^{\theta}=\left\{\left(t_{1}, t_{2}, t_{3}\right)\right\}$.

The set of $\theta$-equivalence classes in $D$ is denoted by $D^{\theta}$. It is of the same cardinality as the set $X$ and thus there is a bijective mapping $\psi: D^{\theta} \rightarrow X$. Define a ternary operation [ ] $]^{\prime}$ on $H$ by: for any $t_{1}, t_{2}, t_{3} \in H$,

$$
\left[t_{1} t_{2} t_{3}\right]^{\prime}=\left\{\begin{array}{l}
{\left[t_{1} t_{2} t_{3}\right]^{*}, \text { if } \operatorname{var}\left(t_{1}\right)=\operatorname{var}\left(t_{2}\right)=\operatorname{var}\left(t_{3}\right)} \\
\psi\left(\left(t_{1}, t_{2}, t_{3}\right)^{\theta}\right), \text { if } \operatorname{var}\left(t_{i}\right) \neq \operatorname{var}\left(t_{j}\right), \text { for some pair } i, j \in\{1,2,3\} .
\end{array}\right.
$$

It is clear by (4) that $\boldsymbol{H}=\left(H,[]^{\prime}\right)$ is a ternary groupoid and $\boldsymbol{H} \in \mathcal{P}_{s c}$. Therefore, the condition (I0) of Def.4 is satisfied. The conditions (I1) and (I2) of Def.4 are fulfilled by Lemma 2 a) and b), c) respectively.

Let $t \in H \backslash X$, i.e. $t=\left[t_{1} t_{2} t_{3}\right]^{\prime}$. (The element $t$ is not primitive in $\boldsymbol{H}$, because if it would be, we would obtain that $\operatorname{var}\left(t_{i}\right) \neq \operatorname{var}\left(t_{j}\right)$, for some pair $i, j \in\{1,2,3\}$ and thus $t=\psi\left(\left(t_{1}, t_{2}, t_{3}\right)^{\theta}\right) \in X$, a contradiction.) Then $t$ is a potent element in $\boldsymbol{H}$ and thus $\underline{t}_{1}=\underline{t}_{2}=\underline{t_{3}}$, i.e. $\operatorname{var}\left(t_{1}\right)=$ $\operatorname{var}\left(t_{2}\right)=\operatorname{var}\left(t_{3}\right)$ and $t=\left[t_{1} t_{2} t_{3}\right]^{*}$. If $t_{1}^{\sim}=t_{3}^{\sim}$, then $t_{1}=t_{3}$ and so $t=\left[t_{1} t_{2} t_{1}\right]^{\prime}$, i.e. $\left(t_{1}, t_{2}, t_{1}\right)$ is the unique pair of divisors of $t$ in $\boldsymbol{H}$. If $t_{1}^{\sim} \neq t_{3}^{\sim}$, then $t=\left[t_{1} t_{2} t_{3}\right]^{\prime}=\left[t_{1} t_{2} t_{3}\right]^{*}=\left[t_{3} t_{2} t_{1}\right]^{*}=\left[t_{3} t_{2} t_{1}\right]^{\prime}$, since $\left[t_{1} t_{2} t_{3}\right]^{\prime}$, $\left[t_{3} t_{2} t_{1}\right]^{\prime} \in\langle t\rangle_{*} \in \mathcal{P}_{s c}$. Hence, the condition (I3) from Def.4 is satisfied. Thus the ternary groupoid $\boldsymbol{H}$ is $\mathcal{P}_{s c}$-injective.

Since $\psi: D^{\theta} \rightarrow X$ is a bijective mapping, it follows that the set $X \backslash i m \psi=$ $\varnothing$, i.e. the set of primes in $\left(H,[]^{\prime}\right)$ is empty. By Bruck theorem for $\mathcal{P}_{s c}$ it follows that the ternary groupoid $\left(H,[]^{\prime}\right)$ is not $\mathcal{P}_{s c}$-free. Thus we have proved the following 
Proposition 9. The class of $\mathcal{P}_{s c}$-free ternary groupoids is a proper subclass of the class of $\mathcal{P}_{\text {sc-injective ternary groupoids. }}$

Hence, the characterization of $\mathcal{P}_{s c}$-free objects by means of $\mathcal{P}_{s c}$-injective ternary groupoids is completed.

\section{REFERENCES}

[1] A. Albert, Power-associative rings, Transactions of the American Mathematical Society, 64 (3) (1948) 552-593.

[2] A. V. Artamonov, Svobodnie n-gruppy, Matem. zametki, 8(4) (1970), 499-507 (in Russian).

[3] A. Borowiec, W. A. Dudek, S. A. Duplij, Basic Concepts of Ternary Hopf Algebras, Journal of Kharkov National University, ser. Nuclei, Particles and Fields, 529, N 3(15) (2001) 21-29.

[4] A. Borowiec, W. A. Dudek, S. Duplij, Bi-element Representations of Ternary groups, Communications in Algebra 34: (2006), 1651-1670.

[5] Bruck R. H., A Survay of Binary Systems, Springer-Verlag, Berlin-GötingenHeidelberg, 1958.

[6] V. Celakoska-Jordanova, Free Power-Commutative Groupoids, Math. Slovaca, 65 (1) (2015), 23-32.

[7] V. Celakoska-Jordanova, Cyclic subgroupoids of an absolutely free groupoid, Proceed. of the III Congress of Math. and Inf. of Macedonia, Struga, 2005 (2007), 217-224.

[8] V. Celakoska-Jordanova, Ternary groupoid powers, Mat. Bilten, 33 (2009), 15-19.

[9] V. Celakoska, Free Ternary Semicommutative Groupoids, Proceed. of the International Scientific Conference, FMNS-2013, Blagoevgrad, Bulgaria, 1 (Math. \& Inf.) (2013), 33-39.

[10] V. Celakoska-Jordanova, E. Celakoska, Canonical commutative ternary groupoids, International Journal of Algebra, 11(1) (2017), 35-42.

[11] V. Celakoska-Jordanova, V. Miovska, Free power-associative n-ary groupoids, (accepted in Math. Slovaca).

[12] Ǵ. Čupona, N. Celakoski, S. Ilić, On Monoassociative Groupoids, Mat. Bilten, 26 (2002), 5-16.

[13] Ǵ. Čupona, N. Celakoski, S. Markovski, D. Dimovski, Vector Valued Groupoids, Semigroups and Groups, in Vector Valued Groupoids, Semigroups and Groups, Maced. Acad. of Sci. and Arts, (1988) 1-78.

[14] S. S. Davidov, Free Commutative Medial Ternary Groupoids, Discrete Math. Appl., 25(4) (2015), 203-210; originally published in Diskretnaya Matematika 27(1) (2015), 34-43 (in Russian).

[15] S. Duplij, Ternary Hopf Algebas, Proceed. of Inst. of Math. of NAS of Ukraine, 43(2) (2002), 439-448.

[16] J. Ježek, Universal Algebra, 2008.

[17] R. Kerner, Ternary algebraic structures and their applications in physics, in the Proc. BTLP, 23rd International Conference on Group Theoretical Methods in Physics, Dubna, Russia (2000); arXiv:math-ph/0011023v1

[18] R. Kerner, The cubic chessboard: geometry and physics, Class Quantum Gravity 14 (1997), A203-A225. 
[19] D. H. Lehmer, A ternary analogue of abelian groups, Amer. J. Math., 54 (2) (1932), 329-338.

[20] Z. Oziewicz, Ternary relative velocity, Astonishing conflict of the Lorentz group with relativity, Physical Interpretations of Relativity Theory, (2007), 292-303.

[21] M. S. Pop, A. Pop, On some relations on n-monoids, Carpathian J. Math., 20 (1) (2004), 87-94.

[22] M. Rausch de Traubenberg, Ternary algebras and groups, Journal of Physics: Conference Series 128 (2008) 012060.

[23] S. A. Rusakov, Algebraicheskie n-arnye sistemy, Navuka i Tehnika, Minsk, 1992 (in Russian).

[24] N. A. Shchuchkin, The Free Abelian n-ary Groups, Chebyshevskii Sb., 12 (2) (2011), $163-170$.

"Ss.Cyril and Methodius" University,

Faculty of Natural Sciences and Mathematics,

Arhimedova 3, 1000 Skopje

R. Macedonia

Email address: vesnacj@pmf.ukim.mk, celakoska@gmail.com

"Ss.Cyril and Methodius" University,

FaCUlty of Mechanical EngineERING,

KARPoŠ II BB, 1000 SKOPJE

R. Macedonia

Email address: emilija.celakoska@mf.edu.mk

"Ss.Cyril And Methodius" University,

Faculty of Natural Sciences and Mathematics,

Arhimedova 3, 1000 Skopje

R. Macedonia

Email address: miovska@pmf.ukim.mk 